\title{
A comparison of the core exciton and nitrogen donor in diamond
}

\author{
Alison Mainwood $\uparrow$ and A M Stoneham $\ddagger$ \\ $\dagger$ Physics Department, King's College London, Strand, London WC2R 2LS, UK \\ $\ddagger$ AEA Technology, Harwell, Didcot, Oxfordshire OX11 ORA, UK
}

Received 13 March 1994

\begin{abstract}
We use molecular dynamics with self-consistent quantum chemistry to compare the dynamics of the relaxation processes around the core exciton in diamond with the properties of the nitrogen substitutional atom, which has the same valence electron configuration. Both show a substantial $\{111\}$ relaxation, associated with the population of an anti-bonding orbital with a neighbouring carbon and not a Jahn-Teller effect. The relaxation into this stable state has no energy barrier in either case, and excites modes of vibration in the frequency range 50-75 meV, with a possible local mode for the core exciton. The lineshapes for the absorption and emission processes in the creation and recombination of the core exciton are obtained from the dynamic calculation.
\end{abstract}

\section{Introduction}

The core exciton was first observed in diamond by Morar and co-workers [1] who measured its binding energy to be $0.19 \pm 0.015 \mathrm{eV}$, and showed that a simple effective mass theory [2] predicted almost exactly this value. In other semiconductors the agreement has not been so good, and it is not at all clear why effective mass theory appears to be applicable for the core exciton while for conventional donors it gives very poor results in diamond. $\mathrm{X} \alpha$ calculations [3] compared the core-exciton with the isocoric donor-nitrogen. This work also suggested that the exciton wavefunction was quite delocalized-about $60 \%$ on the second neighbours to the exciton-as one would expect for an effective-mass-like donor. Since that apparently satisfactory interpretation, the picture has been complicated by several contradictary results. Calculations using local density theory, by Jackson and Pederson [4], predicted a deep s-like state with a binding energy of $0.8-1.7 \mathrm{eV}$ to which transitions from the core hole $1 \mathrm{~s}$ state would be dipole forbidden. They also found a shallow p-like state with a binding energy of about $0.2 \mathrm{eV}$ which would give the core exciton properties described by Morar. They compared the deep state with nitrogen, which is observed to have a $1.7 \mathrm{eV}$ ionization energy [5] in diamond. The evidence for this deep state mounted when Nithianandam [6], using a combination of experimental techniques, derived a binding energy of $1.25 \pm 0.15 \mathrm{eV}$, but found no evidence for the p-like state; however, because of a possible misidentification of the conduction band edge in the earlier work, he argued that this actually corresponds in energy to the value of Morar and co-workers. Batson [7], using symmetry-selected electron energy loss scattering, was able to distinguish the parity of the observed state, and found no evidence for the deep s- or p-like excitons, taking the theory back to the diffuse effective-mass-like state of Morar and co-workers. 
Up to then, although most workers had commented on the parallel between the core exciton and the nitrogen donor, none had investigated the most obvious property of nitrogen-the substantial (111) distortion [8], which might be expected also to occur for the core exciton. This property used to be attributed to a large static Jahn-Teller effect deriving from a partially occupied $T_{2}$ electronic level in the band gap [9]. However, more recent calculations $[10,11]$ do not support this interpretation, showing that the partially occupied level has $A_{1}$ symmetry, and could therefore not give rise to a Jahn-Teller effect. The strong anti-bonding nature of the bond between one of the neighbouring carbon atoms and the nitrogen gives an elongation of that bond and accounts for the (111) distortion observed. Briddon and Jones [11] showed that a local mode vibration with an energy of $166.6 \mathrm{meV}$ (1344 $\mathrm{cm}^{-1}$ ) was associated with the transverse vibration of the unique carbon neighbour to the nitrogen in accordance with previously puzzling isotope data [12]. They also showed [11] that the 1111$\rangle$ vibration of the nitrogen atom, along the direction of its relaxation, has an energy of only $60-110 \mathrm{meV}\left(500-900 \mathrm{~cm}^{-1}\right)$.

$\mathrm{Ma}$ et al [13] attempted to investigate this parallel with the core exciton, although they tried to relate it to the discredited Jahn-Teller explanation. They estimated that a relaxation of approximately $0.2 \AA$ along the (1II) direction corresponded to about $8 \times 160 \mathrm{meV}$ phonons, increasing the binding energy of the exciton from 0.2 to $1.5 \mathrm{eV}$-close to the nitrogen's energy of $1.7 \mathrm{eV}$. They observed a broad tail in the spectrum, which they interpreted as the phonon side band of the transition. The choice of $160 \mathrm{meV}$ for the phonon frequency appears to be a guess based on the Raman frequency in diamond of $165 \mathrm{meV}$, rather than the $166.6 \mathrm{meV}$ local mode. Nonetheless, Briddon's much lower values for the relevant vibration frequency [11] cast some doubt on this energy. The large relaxation suggests that there may be anharmonic effects in the relaxation not considered by Ma et al [13].

We have used the self-consistent molecular dynamics approach described in section 2 to calculate the static properties of the core exciton and nitrogen in diamond (section 3 ). Allowing the relaxation to take place dynamically, we can follow the process to see directly both the vibrational modes of the individual atoms which are excited by the relaxation, and the kinetic and potential energies as functions of time (in section 4). Interpretation of the latter allow us to predict lineshapes in the creation and recombination processes for the core exciton (section 5).

\section{Theoretical approach}

We have used a self-consistent semi-empirical molecular orbital technique, CNDO [14], incorporating Car-Parrinello-like dynamics $[15,16]$ to compare the vibronic properties of nitrogen and the core exciton in diamond. This technique, implemented in the Harwell CHEMOS code, has been applied to nitrogen in diamond before $[17,18,19]$ with good agreement with experimental data. The calculations have been done on clusters of 59 carbon atoms with the outer shell consisting of only the $\mathrm{sp}^{3}$ hybrids directed into the cluster to eliminate dangling bonds. The core exciton has been modelled by giving one carbon atom an additional core charge and by introducing an additional electron into the cluster but retaining the other carbon properties. The empirical parameters used for carbon [20] and nitrogen [17] are those used previously. 


\section{Structure}

As expected, the equilibrium structures of the core exciton and the nitrogen substitutional atom are very similar. In each case there is a substantial relaxation along a $\langle 111\rangle$ direction in which the core exciton atom (or nitrogen) moves away from one of its neighbours towards the plane of the other three. In table 1, the bond lengths are compared. If the carbon atom with a core exciton is denoted $\mathrm{C}^{x}$ in an $n$-atom cluster $\mathrm{C}_{n}$, the binding energy of the core exciton, in the relaxed configuration, is given by

$$
E\left(C_{n-1} C^{x}\right)-E\left(C_{n}^{+}\right)-E\left(C_{n}^{-}\right)+E\left(C_{n}\right)
$$

and it is found to be $2.5 \mathrm{eV}$.

Table 1. Geometry of core exciton and nitrogen defects in $A$. C denotes the unique carbon neighbour, $\mathrm{C}_{3}$ the other carbon neighbours. The interatomic spacing in the perfect diamond crystal is $1.54 \AA$.

\begin{tabular}{lllll}
\hline $\mathrm{X}$ & $\mathrm{X}$ along $\langle 111\rangle$ & $\mathrm{C}$ along $\{111\rangle$ & $\mathrm{C}-\mathrm{X}$ & $\mathrm{X}-\mathrm{C}_{3}$ \\
\hline Core exciton & 0.12 & 0.23 & 1.89 & 1.54 \\
Nitrogen & 0.16 & 0.21 & 1.92 & 1.48 \\
\hline
\end{tabular}

We can assume that when the core hole is produced, the carbon atom on which it is localized will be close to its normal lattice position, and it is this structure which is relevant to the absorption studies. The core exciton would be expected to relax and be vibrating around its equilibrium position within $10^{-10} \mathrm{~s}$ of its creation. Once it is created, the core exciton survives for $10^{-9}-10^{-2} s[21]$ before recombination, a significantly longer time. We can be confident that the excitons observed by emission are in their relaxed structures. The relaxation energy of the core exciton was found to be $1.12 \mathrm{eV}$, slightly larger than the value for nitrogen of $1.05 \mathrm{eV}$. The relaxation of the core exciton and the surrounding atoms contributes nearly half of the binding energy of the exciton.

\section{Relaxation}

The dynamics of the relaxation can be studied in detail. The vibration of the atom with the core exciton and that of its neighbour, resolved along and perpendicular to the axis between them, are shown in figure 1 . The relaxation clearly sets up substantial vibration of both atoms. The frequencies of vibration are all around 50-60 meV, except the $\langle 111\rangle$ core exciton atom, where the frequency is about $160 \mathrm{meV}$ (see table 2). The absolute values of vibrational frequencies calculated in this way are not always very reliable-Wallace et al [16] found that they usually exceeded the experimental values by a factor of 1.5-2.0 for organic molecules in a systematic way, although the parameters used here are not the same as those used in [16].

Another method of calculating the frequencies of vibration of the defect atoms is to displace each in turn a small distance from its equilibrium position and calculate the energy of the cluster allowing all others to relax. This looks at parts of the force constant matrix rather than the vibrations of the cluster as a whole. From these data the dynamic equations can be solved. If the calculation is restricted to only the core exciton atom and its unique carbon neighbour, the frequencies are $45-65 \mathrm{meV}$, except for the transverse vibration of the carbon neighbour of about $120 \mathrm{meV}$ (table 3). We must emphasize that these two methods of calculation are not equivalent. The modes vibrating in the first are those excited by the 


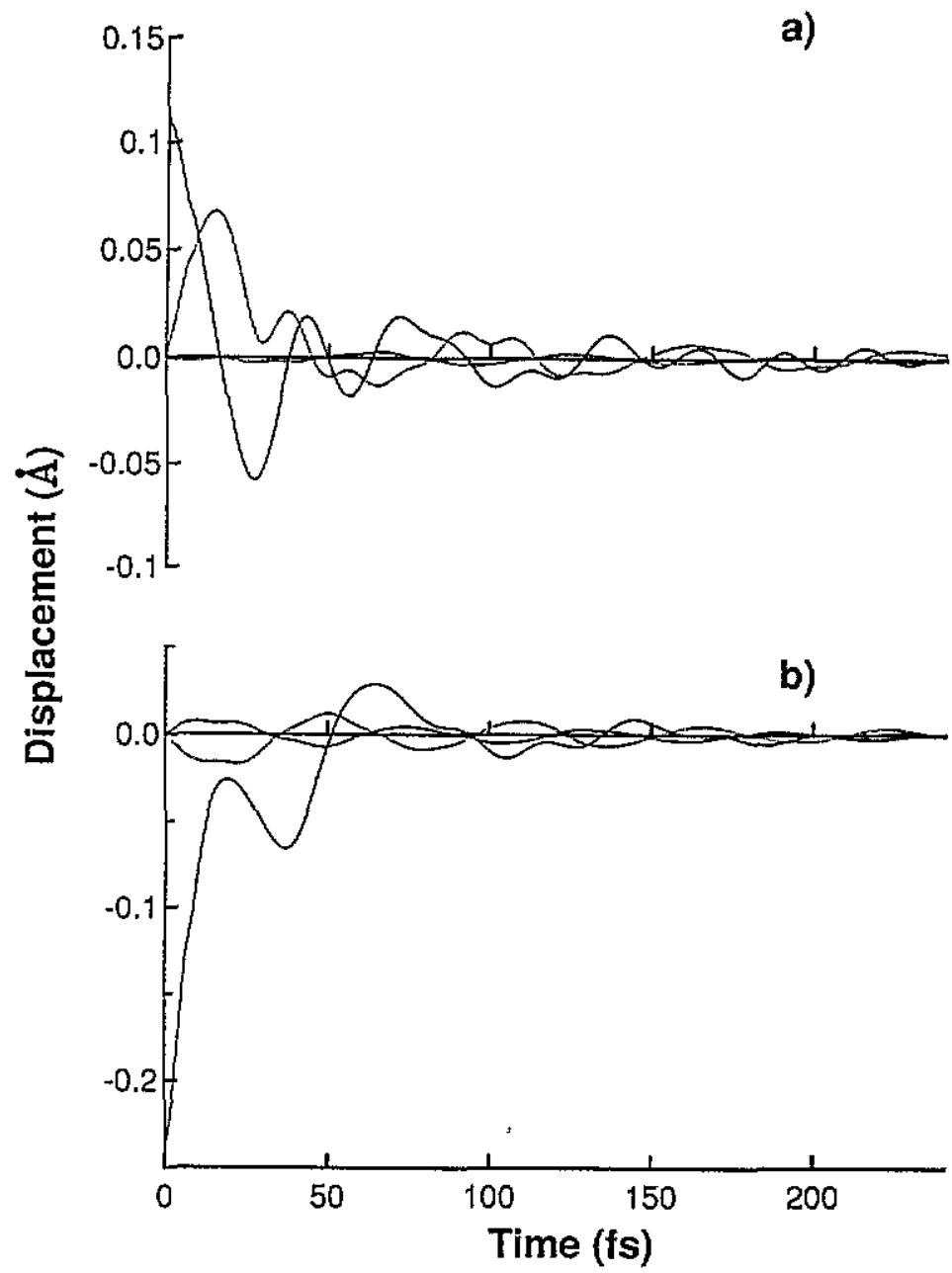

Figure 1. The displacement of (a) the core exciton atom and (b) its unique carbon neighbour resolved parallel (solid line) and perpendicular (dashed lines) to the $\{111\rangle$ direction as they relax into the equilibrium structure for the core exciton. The origin of the displacement is at the equilibrium position of the atoms.

Table 2. The vibrational frequencies associated with the core exciton, nitrogen and the displaced central carbon atom in a perfect cluster, excited by the relaxation to the equilibrium structure. All are in $\mathrm{meV}( \pm 10 \mathrm{meV})$. It is probable that the $160 \mathrm{meV}$ frequency of the core exciton corresponds to a weakly localized mode, as explained in the text.

\begin{tabular}{llll}
\hline$X$ & Core exciton & Nitrogen & Carbon \\
\hline$X \|\langle 111\}$ & 160 & 65 & 50,160 \\
$X \perp\{111\}$ & 60 & 65 & - \\
$C \|\langle 111\rangle$ & 50 & 65 & 65,150 \\
$C \perp\langle 111\}$ & 50 & 75 & - \\
\hline
\end{tabular}

relaxation, some of them strongly anharmonic, perhaps involving carbon atoms which are barely involved in the harmonic vibrations calculated in the second, which is only concerned 
with the curvature of the potential surface very close to equilibrium.

Table 3. The vibrational frequencies from the potential surface very close to equilibrium (a) by these calculations, (b) from Briddon et al [11], and (c) compared to experimental data interpreted by Briddon et al.

\begin{tabular}{lcccl}
\hline & Core exciton & \multicolumn{3}{c}{ Nitrogen } \\
\cline { 2 - 5 } $\mathrm{X}$ & (a) & (a) & (b) & (c) \\
\hline $\mathrm{X} \|\{111\rangle$ & 65 & 60 & $60-110$ & - \\
$\mathrm{X} \perp\langle 111\rangle$ & 40 & 80 & 134 & 140 \\
$\mathrm{C} \|\langle 111\rangle$ & - & - & $60-110$ & - \\
$\mathrm{C} \perp\langle 111\rangle$ & 120 & 116 & 165 & 166.6 \\
\hline
\end{tabular}

We can compare these dynamics with the similar nitrogen case, of which the vibrational frequencies are much better understood [11]. The vibration of the nitrogen atom and carbon neighbour are shown in figure 2. Here, the frequencies of vibration are all in the range 65-75 meV (table 2). However, the second method corresponds more closely to the more sophisticated approach of Briddon et al where the vibration around the nitrogen's equilibrium position, rather than the relaxation into it, was the important property. The frequencies (see table 3) are $60-80 \mathrm{meV}$, corresponding to the values of Briddon et al of 60-110 meV. The carbon neighbour's transverse vibration (probably not induced in figure 2) has a frequency of about $116 \mathrm{meV}$ compared to the $160 \mathrm{meV}$ of Briddon et al. The values of Briddon et al agree well with the available experimental data, so we see that our method is producing rather low values of vibrational frequencies when calculated in this way.

We can conclude that the core exciton has a local mode associated with the (11) relaxation which does not correspond to anything seen in the nitrogen vibration, with an energy above $160 \mathrm{meV}$. This mode (weakly localized because it lies near or only just above the Raman frequency) involves further neighbours to the core exciton atom and therefore is not seen in the dynamic equation calculation. However this calculation shows the transverse vibration of the carbon neighbour of $120 \mathrm{meV}$ (which may be seen as another local mode above $165 \mathrm{meV}$, corresponding to the nitrogen $166.6 \mathrm{meV}$ local mode), but this mode does not seem to be excited by the relaxation.

If the core exciton recombines, it leaves a normal carbon atom displaced at the geometry of the initial state, and the atoms will relax back to their normal positions and vibrate around them. It is this vibration which is seen in the emission spectra. This is shown in figure 3 , and the frequencies are listed in table 2. The magnitude of the relaxation energy is $2.3 \mathrm{eV}$. Note that no transverse modes are excited by this relaxation.

\section{Lineshapes}

The potential energy profiles as the relaxations take place give us a better idea of the vibrations excited in the defect and its surroundings than the simple harmonic approximations which are usually assumed. The relaxations are quite large, up to $15 \%$ of the bond lengths, and we would expect anharmonic effects to be significant. Figure 4 shows the relaxations for the three systems that we are considering as functions of time. Each shows a high-frequency vibration which is not associated with the defect (compare with the defect atom vibrations in figures $1-3$ ), and seems to be a vibration at the cluster 


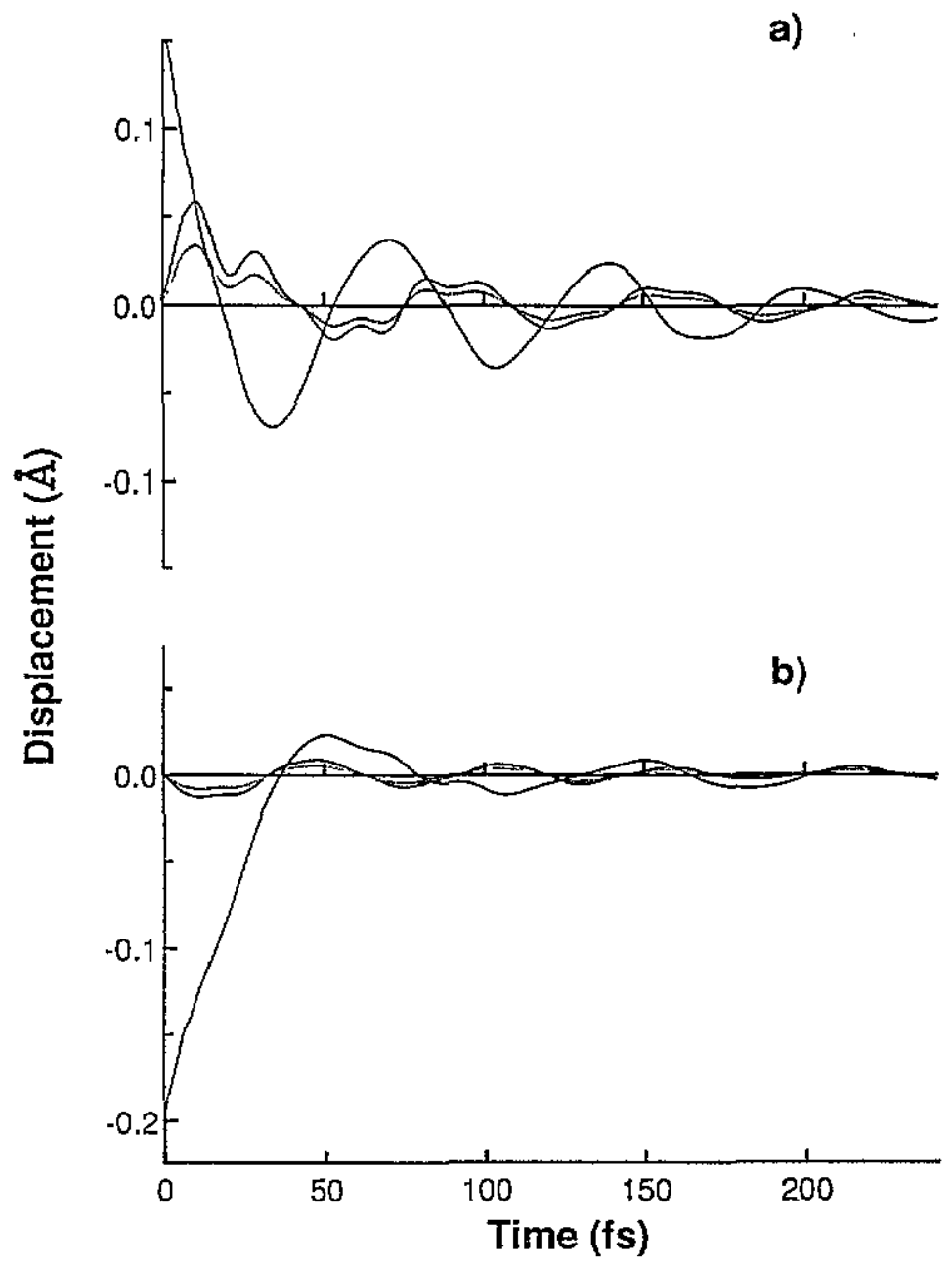

Figure 2. Similar to figure 1, for the nitrogen atom and its carbon neighbour.

surface. The frequencies of vibration associated with the relaxation of the defects can be obtained from these energy profiles by taking their Fourier transforms.

Consider a single harmonic oscillator, with frequency $\omega_{0}$. If the oscillator is displaced from equilibrium until its potential energy is $E_{0}$ and then released, its potential energy $E(t)$ will vary with time as

$$
E(t)=E_{0} \mathrm{e}^{2 \mathrm{i} \omega_{0} t}
$$

The Fourier transform of this, $P(\omega)$, is

$$
P(\omega)=E_{0} \delta\left(\omega-2 \omega_{0}\right)
$$

For this system, with only one harmonic mode, the function $A(\omega)$ which generates the lineshape function $G(\omega)$ is (see the appendix)

$$
A(\omega)=\delta\left(\omega-\omega_{0}\right)-\delta\left(\omega+\omega_{0}\right)
$$




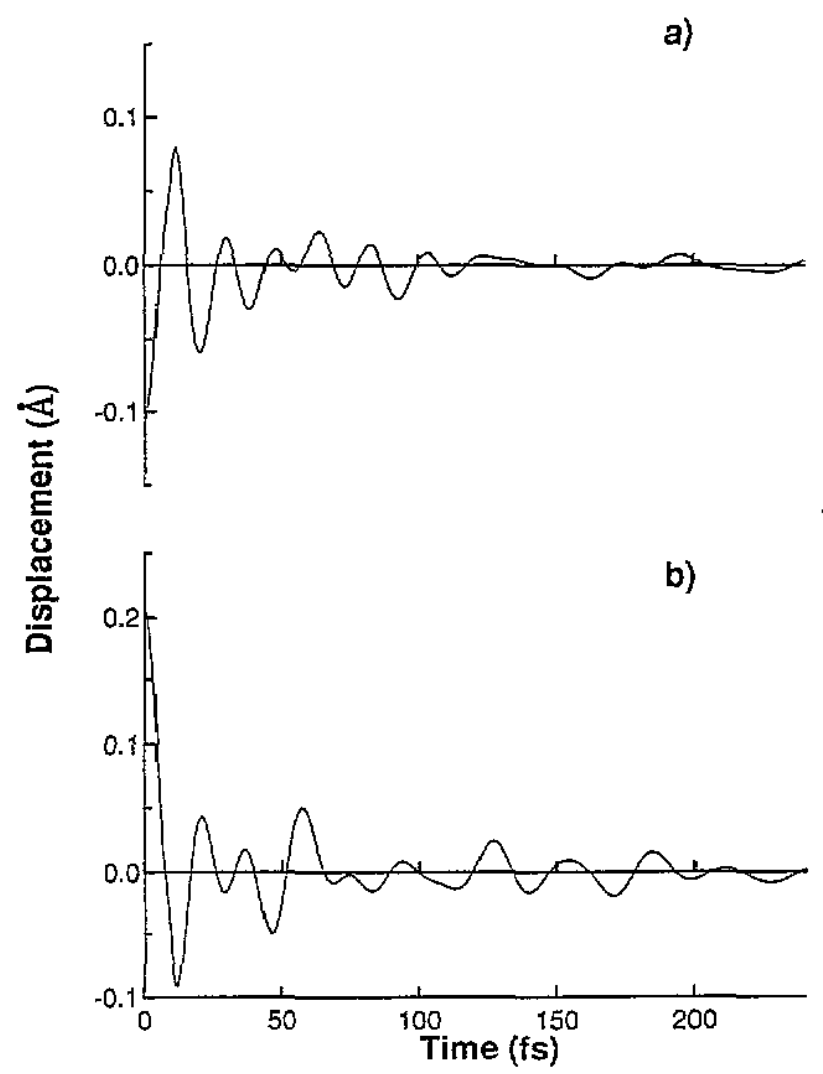

Figure 3. Similar to figure 1 for the carbon atom and its neighbour, relaxing back to their normal positions from the core exciton structure after the exciton recombines.

where the - sign is a construction to make $A(\omega)$ antisymmetric, and the Huang-Rhys factor $S_{0}$ is

$$
S=\frac{E_{0}}{\hbar \omega_{0}} .
$$

The resemblance between equations 5.2 and 5.3 makes it possible to see how the lineshape function, $G(\omega)$, can be calculated from the potential energy of the defect as it relaxes into its equilibrium state. The only assumption is that the vibrations are harmonic.

A sum of harmonic oscillators, corresponding to normal modes $\omega_{n}$ around the defect, generates a simple sum of the components in (5.2) weighted by $E_{n}$

$$
E_{0}=\sum_{n} E_{n}
$$

such that

$$
A(\omega)=\sum_{n} \frac{E_{n}}{E_{0}}\left[\delta\left(\omega-\omega_{n}\right)-\delta\left(\omega+\omega_{n}\right)\right]
$$

will generate the resultant lineshape function.

The vibrations shown in figure 4 clearly include some transient modes and some which are more persistent as well as a slow overall energy convergence. If each mode $n$ decays 


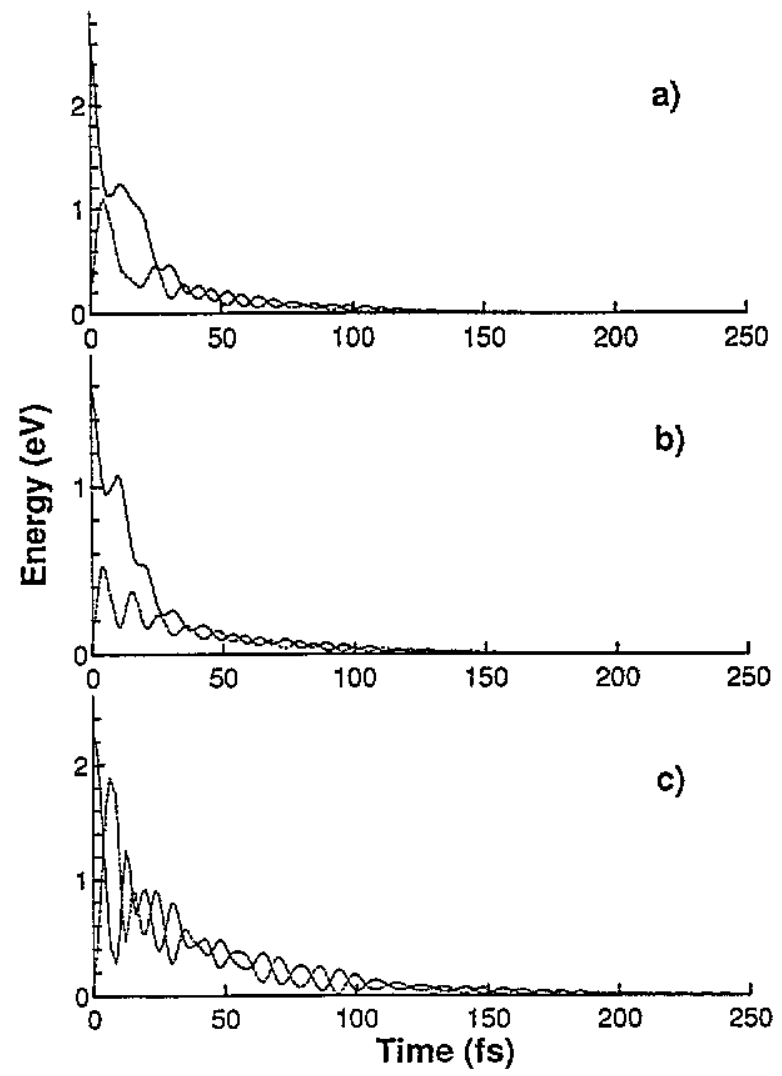

Figure 4. The kinetic energy (dashed line) and potential energy (solid line) as (a) the core exciton, (b) the nitrogen atom and (c) the carbon atom relax into their equilibrium structures.

by $\exp \left(-\alpha_{n} t\right)$ then

$$
E(t)=\sum_{n} E_{n} \mathrm{e}^{-2 \alpha_{n} t} \mathrm{e}^{2 \mathrm{i} \omega_{n} t}
$$

and $P(\omega)$ contains a sum of $n$ Lorentzian peaks broadened by $2 \alpha_{n}$,

$$
P(\omega)=\sum_{n} \frac{\alpha_{n} E_{n}}{4 \alpha_{n}^{2}+\left(2 \omega_{n}-\omega\right)^{2}}
$$

and, by comparison, the lineshape function becomes

$$
A(\omega)=\sum_{n}\left[\frac{2 \alpha_{n} E_{n}}{\alpha_{n}^{2}+\left(\omega_{n}-\omega\right)^{2}}-\frac{2 \alpha_{n} E_{n}}{\alpha_{n}^{2}+\left(\omega_{n}+\omega\right)^{2}}\right] .
$$

The relaxation energy $E_{0}$ is distributed across the modes in the ratio of the $E_{n}$, so the Huang-Rhys factor $S_{n}$ for the $n$th mode is

$$
S_{n}=\frac{E_{n}}{\hbar \omega_{n}} \text {. }
$$

We have taken the Fourier transforms of the potential energy in the relaxation to identify the frequencies of the modes, and from them constructed the $A(\omega)$ from which the lineshapes have been calculated. It is not clear over what time period the Fourier transforms should be taken. We are limited by the resolution of the numerical Fourier transform process, 
and the consideration that the optical processes occur over a few tens of femto seconds. We compared the Fourier transforms taken over 62, 124 and 248 fs from the start of the relaxation, and some taken after a short time delay, and found that they differed very little. Those taken over the full period shown in figure 5 , which had the highest resolution, were used. The high-energy vibrations, which appear to be artifacts of the clusters, have been subtracted from the frequency distributions. The theory used to construct these lineshapes is taken from [22] and is explained in detail in the appendix. The vibronic lineshape for absorption-the perfect diamond lattice relaxing into the core exciton structure when it is created-is predicted in this way to be of the shape shown in figure 5. The equivalent lineshape for emission-when the core exciton recombines and the lattice relaxes back to the normal diamond structure-is shown in figure 6. Similar calculations are quite possible for the nitrogen relaxation, but do not correspond to any observed bandshapes.

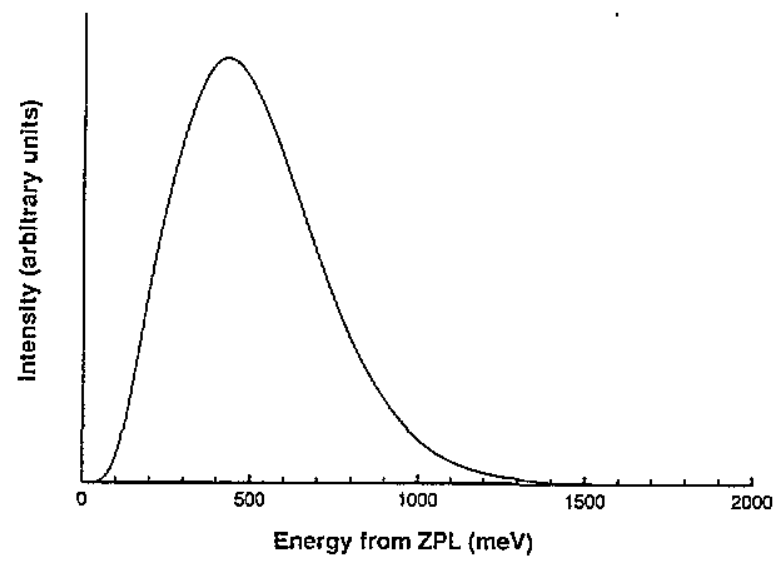

Figure 5. The predicted lineshape for absorption of $\mathrm{x}$-rays in the formation of a core exciton in diamond.

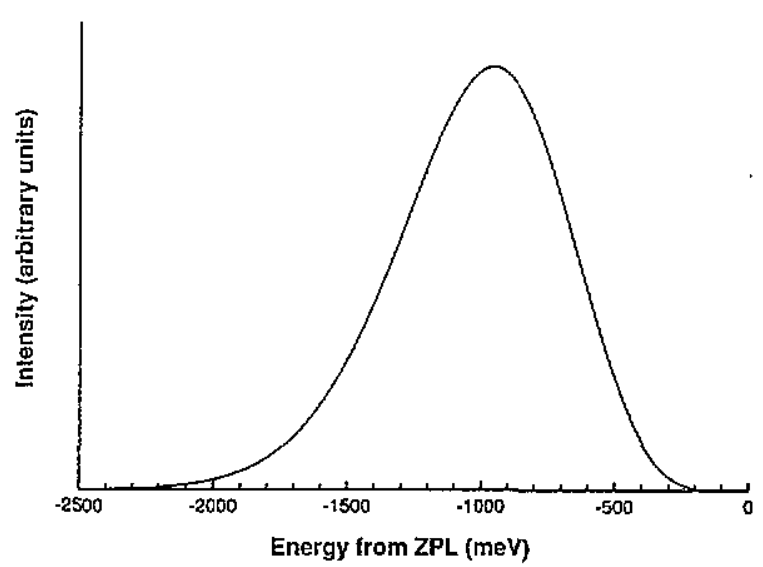

Figure 6. The predicted lineshape for emission of $x$-rays when the core exciton recombines in diamond.

Because the Huang-Rhys factors are very large, 17 and 30 respectively, there are no zero-phonon lines. The lineshapes shown correspond to a sum of convolutions of the onephonon bandshape $\Gamma_{1}(\omega)$ with itself many times, so the resultant lineshape is quite close 
to a Gaussian, and has very little dependence on the form of $\Gamma_{1}(\omega)$. The Stokes shift is the sum of the shifts of the peaks in absorption and emission from the zero-phonon line, and is calculated here as $1.34 \mathrm{eV}$, which compares with that reported by $\mathrm{Ma}$ et al [13] of about $0.6 \mathrm{eV}$. In a similar way, the linewidths calculated here are 460 and $750 \mathrm{meV}$ in absorption and emission-again larger than the experimental absorption value of about $200 \mathrm{meV}$ [13]. According to Huang-Rhys theory, the shift of the peak and its linewidth are approximately proportional to the Huang-Rhys factor and its square root respectively [22]. From the experimental data on the Stokes shift and linewidth, we would expect the relaxation energies to be $0.4-1 \mathrm{eV}$, rather than the 1.1 and $2.3 \mathrm{eV}$ that we find.

\section{Conclusions}

We have shown that the properties of the core exciton are very similar in many respects to the isocoric donor-nitrogen. Both have substantial relaxations, with no energy barrier, away from one of their carbon neighbours. They have very similar modes of vibration around their equilibrium positions. However it is not necessarily these modes which are excited by the relaxations, and the tranverse vibration of the carbon neighbour, which is seen as a local mode associated with nitrogen at $166.6 \mathrm{meV}\left(1344 \mathrm{~cm}^{-1}\right)$, and has an analogy in the core exciton case, is not seen in the relaxation. On the other hand, a local mode associated with the core exciton's relaxation, which has no analogy in nitrogen's vibration, plays a prominent part in the core exciton's relaxation.

We have shown how dynamics calculations can be used to predict the lineshapes for absorption and emission spectra. The lineshape function that we calculate, $G(\omega)$, has very wide application wherever there is appreciable vibronic coupling, involving either radiative or non-radiative transitions [23].

The core exciton in diamond shows a large electron-lattice coupling which is not associated with a Jahn-Teller effect, and can be modelled well with Franck-Condon coupling theory. Effective mass theory cannot account for many of the observed properties. Qualitatively, the analogy with the nitrogen substitutional atom is a useful one.

\section{Acknowledgments}

We are grateful to Professor Gordon Davies for helpful discussions. One of us (AM) was partly funded by the SERC.

\section{Appendix}

The function $A(\omega)$ is defined by 10.7 .23 of [22] and gives the distribution of the HuangRhys factors $S_{0}$ over modes $\alpha$ :

$$
A(\omega) d \omega=\sum_{\omega<\omega_{\alpha}<\omega \div \mathrm{d} \omega} S_{0 \alpha} / \sum_{\text {all } \alpha} S_{\alpha} .
$$

At low temperatures $(k T \ll \hbar \omega)$, we can follow through [22] to show that

$$
\begin{aligned}
& \zeta(t)=\int_{-\infty}^{\infty} \mathrm{d} \omega^{\prime} A\left(\omega^{\prime}\right) \mathrm{e}^{\mathrm{i} \alpha^{\prime} t} \\
& g(t)=\mathrm{e}^{\mathrm{i} \omega_{t,} t} \mathrm{e}^{\zeta(t)}
\end{aligned}
$$


where $\omega_{i j}$ is the frequency of the electronic transition, giving the lineshape function

$$
G(\omega)=\frac{1}{2 \pi} \int_{-\infty}^{\infty} \mathrm{d} t \mathrm{e}^{-\mathrm{i} \omega t} g(t) .
$$

In [22] it is shown that the exponential in equation (A.3) indicates a sum of convolutions of the one-phonon lineshape $\Gamma_{1}(\omega)$ with itself to form one-, two-, three-, $\ldots$ phonon contributions. At low temperatures $A(\omega)=\Gamma_{1}(\omega)$. Because of the convolution process, if the Huang-Rhys factor is large (relaxation is large), then the zero-phonon line will not be seen, the lineshape will approximate to a Gaussian and the shift of the peak from the zero-phonon energy and linewidth will be independent of the form of $A(\omega)$.

At higher temperatures, the functions are more complex, in particular the function $g(t)$ and $S$, the Huang-Rhys factor, are temperature dependent, but the lineshapes can be calculated in a similar way.

\section{References}

[1] Morar J F, Himpsel F J, Hollinger G, Hughes G and Jordan J L 1985 Phys. Rev. Lett. 541960

[2] Bassani F 1977 Appl. Opt. 94093

[3] Alves H W L, Chacham H, Alves J J A and Leite J R 1988 Solid State Commun. 5495

[4] Jackson K A and Pederson M R 1991 Phys. Rev. Lett. 672521

[5] Farrer R G 1969 Solid State Commun. 7685

[6] Nithianandam J 1992 Phys. Rev. Lett. 693108

[7] Batson P E 1993 Phys. Rev. Lett. 701822

[8] Smith W V, Sorokin P P, Gelles L L and Lasher G J 1959 Phys. Rev. 1151546

Cook R J and Whiffen D J 1966 Proc. R. Soc. A 29599

[9] Messmer R P and Watkins G P 1973 Phys. Rey. B 72568

[10] Bachelet G B, Baraff G A and Schlutter M 1981 Phys. Rev. B 244736

[11] Briddon P R and Jones R 1993 Physica B 185179

[12] Collins A T and Woods G S 1982 J. Phys. D: Appl. Phys. 151431

[13] Ma Y, Skytt P, Wassdahl N, Glans P, Mancini D C, Guo J and Nordgren J 1993 Phys. Rev. Lett. 713725

[14] Pople J A and Beveridge D L 1970 Approximate Molecular Orbital Theory (New York. McGraw-Hill)

[15] Car R and Parrinello 1985 Phys. Rev, Lett. 552471

[16] Wallace D S, Stoneham A M, Hayes W, Fisher A J and Harker A H 1991 J. Phys.: Condens. Matter 33879

[17] Mainwood A 1979 J. Phys. C: Solid State Phys. 122543

[18] Mainwood A 1994 Mater. Sci. Forum 143-147 51

[19] Mainwood A 1994 Phys. Rev. B 497934

[20] Harker A H and Larkuns F P 1979 J. Phys. C: Solid State Phys. 122487

[21] Itoh N 1982 Adv. Phys. 31491

[22] Stoneham A M 1975 Theory of Defects in Solids (Oxford: Oxford University Press)

[23] Stoneham A M 1981 Rep. Prog. Phys. 441251 